\title{
Synthesis of fractal light pulses by quasi-direct space-to-time pulse shaping
}

\author{
Omel Mendoza-Yero, ${ }^{1,2, *}$ Benjamín Alonso, ${ }^{3}$ Gladys Mínguez-Vega, ${ }^{1,2}$ Íñigo Juan Sola, ${ }^{3}$ \\ Jesús Lancis, ${ }^{1,2}$ and Juan A. Monsoriu ${ }^{4}$ \\ ${ }^{1}$ GROC·UJI, Departament de Física, Universitat Jaume I, 12080 Castelló, Spain \\ ${ }^{2}$ Institut de Noves Tecnologies de la Imatge, Universitat Jaume I, 12080 Castelló, Spain \\ ${ }^{3}$ Departamento de Física Aplicada, Universidad de Salamanca E-37008 Salamanca, Spain \\ ${ }^{4}$ Centro de Tecnologías Físicas, Universitat Politècnica de València, E-46022 Valencia, Spain \\ *Corresponding author: omendoza@fca.uji.es
}

Received October 13, 2011; revised January 10, 2012; accepted January 18, 2012; posted January 18, 2012 (Doc. ID 156405); published March 21, 2012

\begin{abstract}
We demonstrated a simple diffractive method to map the self-similar structure shown in squared radial coordinate of any set of circularly symmetric fractal plates into self-similar light pulses in the corresponding temporal domain. The space-to-time mapping of the plates was carried out by means of a kinoform diffractive lens under femtosecond illumination. The spatio-temporal characteristics of the fractal pulses obtained in this way were measured by means of a spectral interferometry technique assisted by a fiber optics coupler (STARFISH). Our proposal allows synthesizing suited sequences of focused fractal femtosecond pulses potentially useful for several current applications, such as femtosecond material processing, atomic, and molecular control of chemical processes or generation of nonlinear effects. (C) 2012 Optical Society of America

OCIS codes: $050.1970,320.5540$.
\end{abstract}

The study of structures that exhibit geometric selfsimilarities has been a matter of scientific concern since the Mandelbrot's work in the 1980s about the fractal geometry of nature [1]. In optics, the first scientific contributions on this topic were addressed to the analysis of light scattered and diffracted by fractal structures usually known as diffractals [2-5]. Here, it should be recalled that self-similarity means that, within a given distribution (i.e., spatial or frequency distribution), some of its parts have the same shape as the whole set. In nonlinear optics the existence of self-similarity distributions has been reported in different physical phenomena. For instance, based on a technique of symmetry reduction, the selfsimilar propagation of parabolic pulses in amplifiers has been corroborated [6-7]. Additionally, the existence of stable pulses that propagate self-similarly in a laser cavity was numerically predicted and later experimentally verified by using a fiber laser [8]. On this subject, an overview of self-similar pulse propagation and its use in the development of high-power ultrafast optical sources, pulse synthesis, and all-optical pulse regeneration has been also published [9].

The above-mentioned self-similarity feature of light pulses is also presented in fractal structures, but this time connected with their spatial structure or diffraction effects on ultrashort pulses as we will see later. With the relatively recent discovery of several practical applications of fractal structures (i.e., for designing microwave antennas with broadband behavior [10], increasing near-field resolution and enhancing transmission with sub-wavelength apertures [11], or constructing novel photonic crystals with potential applications in telecommunication and electro-optical systems [12]), the scientific community's interest in diffractals seems to be growing up. This fact is supported by the introduction of a novel family of diffracting apertures known as fractal zone plates (FZPs) [13]. These apertures, among other applications, have been used in the generation of optical vortices employed for trapping and rotating microparticles $[14,15]$ or as image-forming devices with an extended depth of field and reduced chromatic aberration under white-light illumination [16]. FZPs can be regarded as a set of circularly symmetric apertures with spatial self-similar distribution in the squared radial coordinate $r^{2}$. Consequently, the axial irradiance caused by the diffraction of quasi-monochromatic light by FZPs also exhibits self-similar features. In this case the axial irradiance is mainly determined by the Fourier transform of the pupil function of the FZPs expressed in $r^{2}$. Based on the above idea, but this time using fractal generalized zone plates (FraGZPs), self-similar irradiance patterns generated along the optical axis were reported [17].

In the frequency domain, the self-similarity property has been explored to achieve spectral distributions with fractal properties. Here, taking advantage of the dual role played by the wavelength $\lambda$ and the axial distance $z$ in the Huygens-Fresnel diffraction formula, the self-similar behavior of axial irradiance patterns was mapped to the spectral domain to achieve fractal distribution of frequencies [18]. In this context, the spectrum scalability from the transmission properties of fractal Cantor multilayer dielectric structures was verified [19].

However, as far as we know reports of the self-similar behavior of fractal plates have been limited to the spatial or spectral domains, with no mention of similar effects in the time domain. In this letter we demonstrate that a kinoform diffractive lens (KDL) can be used to generate self-similar light pulses from any set of circularly symmetric fractal plates (i.e., FZPs or FraGZPs). Our proposal is based on the use of a diffractive pulse shaper that was introduced theoretically [20]. It allows mapping the spatial structure of circularly symmetric binary plates into the temporal domain. The experiment was carried out with a technique based on the spatiotemporal amplitude and phase reconstruction by Fourier transform of 
the interference spectra of the optical pulses (STARFISH) [21].

In the present configuration, the shaper is composed of a FZP and a KDL, which are facing each other. The shaper is illuminated with a spatially plane wave and temporally ultrashort light. Its output is located at the focal point of the KDL $\left(z=z_{0}\right)$ for the central wavelength of the pulse $\lambda_{0}$, where the light distribution is both temporally shaped and spatially focused. The temporal window of the shaper $\Delta t$ is given by the expression $\Delta t=a^{2} /\left(2 c z_{0}\right)$, where $c$ denotes the velocity of light in vacuum and $a$ holds for the maximum extension of the FZP. At the focal point $z=z_{0}$, the amplitude of the temporal waveform $u_{\text {out }}(\tau)$ is given approximately by the following convolution expression [20]:

$$
u_{\text {out }}(\tau) \cong u_{0}(\tau) \otimes\left[q(\tau / \Delta t) \exp \left(-i \omega_{0} \tau\right)\right]
$$

In Eq. (1), $u_{0}(\tau)$ is the amplitude of the incident pulse, $q(\varsigma)$ is the transmittance of the mask such that $\varsigma=(r / a)^{2}$ and $\omega_{0}=2 \pi c / \lambda_{0}$. Hence, if we assume a few-cycle incident pulse $\left|u_{\text {out }}(\tau)\right|^{2} \cong|q(\tau / \Delta t)|^{2}$. Under this condition, the spatial features of plates are mapped into the time domain, see Fig. 3(a) and 3(b) in [20].

FZPs are constructed from the fractal structures known as the Cantor set. The construction process is controlled by two fundamental parameters: the generator $N$ and the growing stage $S$. In mathematical terms, the FZP transmittance function, $q(\varsigma)$, developed up to a certain growing stage $S$, can be expressed as [13]

$$
q(\varsigma)=\operatorname{rect}(\varsigma-1 / 2) \prod_{i=0}^{S} \operatorname{rect}\left[\frac{\bmod \left(\varsigma-1+p_{i} / 2, p_{i}\right)}{p_{i}}\right] .
$$

In Eq. $2, p_{i}=2 /(2 N-1)^{i}$, $\operatorname{rect}(x)$ is the rectangle function of argument $x$, whereas $\bmod (x, y)$ gives the remainder on the division of $x$ by $y$. Here, we consider the construction of FZPs defined by the parameters $N=2$, $S=0,1,2$, and $a=4.33 \mathrm{~mm}$. In practice, the FZPs were fabricated by direct laser writing on a chrome photomask using a photolithographic machine. The writing machine allows obtaining photomasks with global resolution of $0.2 \mu \mathrm{m}$ and minimum feature size of $0.8 \mu \mathrm{m}$.

In Fig. 1, the optical setup is shown. We use STARFISH [21] as the spatiotemporal diagnostic tool for the temporally shaped, spatially focused output pulses. Basically, it consists of spatially resolved spectral interferometry using a fiber optic coupler as an interferometer. The arms of the interferometer are composed of two optical fibers of equal length. In the optical setup of Fig. 1, the light coming from a Ti: sapphire laser source that emits $t_{0}=$ 35 fs intensity full-width at half maximum (FWHM) pulses is split in two beams: the reference and the test beam. Before it impinges in the shaper, the test beam is spatially expanded with the help of a $4 \times$ all-mirrors beam expander. At the focus of the KDL, the optical fiber of the test arm spatially scans the pulse in the transverse direction to its propagation axis.

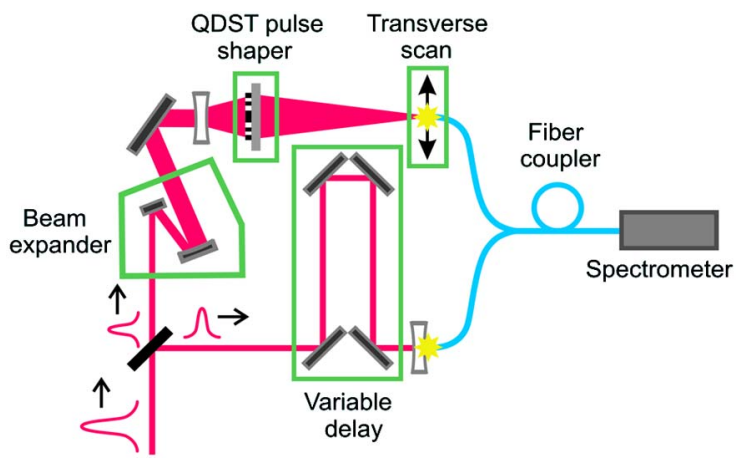

Fig. 1. (Color online) Optical setup. Measurement of temporal-shaped, self-similar ultrashort pulses by using spatially resolved spectral interferometry assisted by a fiber optics coupler.

For our experiment, the KDL has a focal length of $z_{0}=$ $50 \mathrm{~mm}$ for the central wavelength of the pulse $\left(\lambda_{0}=800 \mathrm{~nm}\right)$. Hence, the numerical aperture (NA) at the focal plane $z=z_{0}$ is NA $=0.087$, which is slightly lower than the NA provided by the fiber's manufacturer $(\mathrm{NA}=0.11 \pm 0.01)$. To achieve a better coupling of light into the optical fiber during the scanning process, the NA of the system was further reduced by including a divergent lens $(-40 \mathrm{~mm}$ focal length) a few millimeters before the entrance of the shaper (see Fig. 1). By doing so, the output plane of the optical system was increased until the value $z=200 \mathrm{~mm}$ resulting in the $\mathrm{NA}=0.02$. Note that this value is about 5 times lower than the NA of the optical fiber. The dispersion introduced by the material of the divergent lens is pre-compensated by using dispersion-compensating prism pairs located at the last unit of the femtosecond laser. Because this compensation also affects the light at the reference arm, we added an equal lens just before the entrance of the corresponding optical fiber. The relative delay between the pulses required for the interferometry is controlled by the delay line shown in Fig. 1. For each transverse position, both pulses are combined inside the fiber coupler and leave it through the common port that is directly connected to a standard spectrometer, where the interference spectra are measured. In our experiment, the spectral phase of the reference beam is obtained on-axis just by means of a LX-SPIDER measurement. From the spectral interference, the spectral phase and amplitude at each point are obtained by fringe analysis using Fourier transform algorithms [22].

In Fig. 2, the spatiotemporal characteristics of the measured light together with the corresponding numerical simulations are shown in logarithmic scale. Additionally, in the left part of Fig. 2 , the FZPs used in the experiment are included as insets. For the simulations, the field amplitude at the output plane was determined by means of the generalized Huygens-Fresnel integral for each frequency component of the pulse. Then, the amplitude of temporal waveforms is assessed from the Fourier transform of the field amplitude. From a visual inspection of Fig. 2, one can see that the fractal behavior of the light is mainly concentrated within the spatial interval from $-10 \mu \mathrm{m}$ to $10 \mu \mathrm{m}$. This is consistent with the fact that the working zone of the shaper is expected for points 

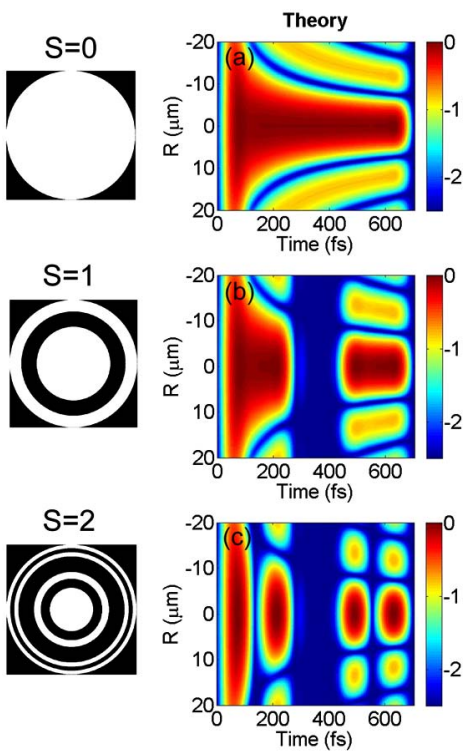
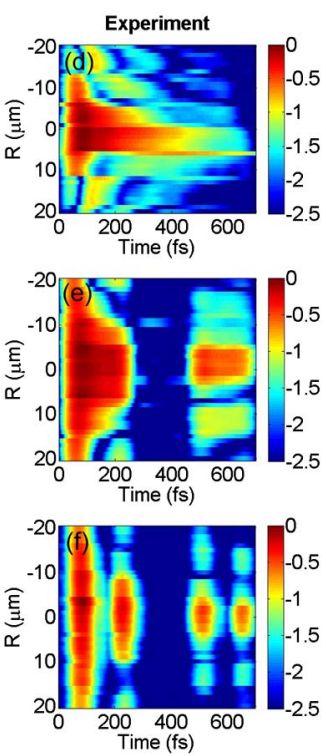

Fig. 2. (Color online) Spatiotemporal characteristics of the synthesized ultrashort fractal pulses. Numerical simulations $(a-c)$ and corresponding experimental results $(d-f)$.The FZPs are included as insets.

in the close vicinity of the optical axis [20], where the previous analysis holds.

However, the extension of the above useful interval depends on the location of the output plane and the spatial structure of the FZP. In Fig. 2, the temporal self-similar property is observed from two characteristic spatiotemporal electric fields with different fractal levels. That is, the spatiotemporal field structure with $S=2$-Fig. 2(c) and $2(\mathrm{f})$ - are modulated versions of the corresponding ones in the previous stage $S=1-$ Fig. 2(b) and 2(e). As expected from the definition of a fractal structure, the synthesized pulses are contained within the same temporal window $\Delta t \cong 625$ fs.

It is clear from Fig. 2 that experimental spatiotemporal electric fields agree quite well with the theoretical results. We believe that small discrepancies observed in Fig. 2 are mainly caused by the deviations of the real pulse from the ideally assumed plane-wave and few-cycle incident pulse. In addition, the convolution of the incident electric field amplitude (having a finite temporal width) with the transmittance function of the mask causes the synthesized pulses to suffer from temporal stretching. The more the width $\delta$ of the transparent zones of the FZP, mapped into the time domain by the KDL, is longer than temporal width $t_{0}$ of the incoming light, the better. The optimum condition for the shaper to carry out the space-to-time mapping is such that the expression $\delta^{2} /\left(2 c z_{0}\right) \gg t_{0}$ is fulfilled in all cases. In our experiment, the term $\delta^{2} /\left(2 c z_{0}\right)$ varies for each FZP, giving 625 fs $(S=0), 208$ fs $(S=1)$ and $70 \mathrm{fs}(S=2)$. Therefore, the near on-axis pulses generated with the FZP with $S=$ 2 in Fig. 2(f) are clearly no longer square but temporally Gaussian-like pulses.

The ability of the pulse shaper to synthesize sequences of fractal pulses at the focus of the KDL might be inter- esting in nonlinear material processing or femtochemistry. In optical wireless communication, fractal modulation by ultra-short wavelet pulse shaping has attracted significant attention as an efficient way to implement multirate communications [23].

This research was funded by the Spanish Ministerio de Ciencia e Innovación (MICINN) and FEDER, through the projects FIS2010-15746, DPI2008-02953, and SAUUL (CSD2007-00013) and the Fundació Caixa Castelló (P11B2010-26). The authors are very grateful to the Serveis Centrals d'Intrumentació Científica of the Universitat Jaume I for the use of the femtosecond laser and the laser writing machine. We also thank Prof. Dr. Jürgen Jahns for the fabrication of the diffractive lenses.

\section{References}

1. B. B. Mandelbrot, The Fractal Geometry of Nature, 2nd ed. (Freeman, 1982).

2. M. V. Berry, J. Phys. A 12, 781 (1979).

3. C. Allain and M. Cloitre, Phys. Rev. A 36, 5751 (1987).

4. D. A. Hamburger-Lidar, Phys. Rev. E 54, 354 (1996).

5. A. D. Jaggard and D. L. Jaggard, J. Opt. Soc. Am. A 15, 1626 (1998).

6. M. E. Fermann, V. I. Kruglov, B. C. Thomsen, J. M. Dudley, and J. D. Harvey, Phys. Rev. Lett. 84, 6010 (2000).

7. V. I. Kruglov, A. C. Peacock, J. D. Harvey, and J. M. Dudley, J. Opt. Soc. Am. B 19, 461 (2002).

8. F. Ö. Ilday, J. R. Buckley, W. G. Clark, and F. W. Wise, Phys. Rev. Lett. 92, 213902 (2004).

9. J. M. Dudley, C. Finot, D. J. Richardson, and G. Millot, Nat Phys. 3, 597 (2007).

10. K. J. Vinoy, K. A. Jose, K. K. Varadan, and V. V. Varadan, Microwave Opt. Technol. Lett. 29, 215 (2001).

11. J. A. Matteo and L. Hesselink, Opt. Express 13, 636 (2005).

12. S. W. Wang, X. Chen, W. Lu, M. Li, and H. Wang, Appl. Phys. Lett. 90, 211113 (2007).

13. G. Saavedra, W. D. Furlan, and J. A. Monsoriu, Opt. Lett. 28, 971 (2003).

14. S. H. Tao, X. C. Yuan, J. Lin, and R. E. Burge, Appl. Phys. Lett. 89, 031105 (2006).

15. W. D. Furlan, F. Giménez, A. Calatayud, and J. A. Monsoriu, Opt. Express 17, 21891 (2009).

16. W. D. Furlan, G. Saavedra, and J. A. Monsoriu, Opt. Lett. 32 , 2109 (2007).

17. O. Mendoza-Yero, M. Fernández-Alonso, G. Mínguez-Vega, J. Lancis, V. Climent, J. A. Monsoriu, W. D. Furlan, F. Giménez, A. Calatayud, and J. A. Monsoriu, J. Opt. Soc. Am. A 26, 1161 (2009).

18. O. Mendoza-Yero, G. Mínguez-Vega, M. Fernández-Alonso, J. Lancis, E. Tajahuerce, V. Climent, and J. A. Monsoriu, Opt. Lett. 34, 560 (2009).

19. A. V. Lavrinenko, S. V. Zhukovsky, K. S. Sandomirski, and S. V. Gaponenko, Phys. Rev. E 65, 036621 (2002).

20. G. Mínguez-Vega, O. Mendoza-Yero, J. Lancis, R. Gisbert, and P. Andrés, Opt. Express 16, 16993 (2008).

21. B. Alonso, Í. J. Sola, Ó. Varela, J. Hernández-Toro, C. Méndez, J. San Román, A. Zaïr, and L. Roso, J. Opt. Soc. Am. B 27, 933 (2010).

22. L. Lepetit, G. Cheriaux, and M. Joffre, J. Opt. Soc. Am. B 12, 2467 (1995).

23. M. Kavehrad and B. Y. Hamzeh, Proc. SPIE 5598, 144 (2004). 